University of Nebraska - Lincoln

DigitalCommons@University of Nebraska - Lincoln

2000

\title{
Optical Communication Networks for the Next-Generation Internet
}

Arun K. Somani

Byrav Ramamurthy

University of Nebraska-Lincoln, bramamurthy2@unl.edu

Follow this and additional works at: https://digitalcommons.unl.edu/csearticles

Part of the Computer Sciences Commons

Somani, Arun K. and Ramamurthy, Byrav, "Optical Communication Networks for the Next-Generation Internet" (2000). CSE Journal Articles. 77.

https://digitalcommons.unl.edu/csearticles/77

This Article is brought to you for free and open access by the Computer Science and Engineering, Department of at DigitalCommons@University of Nebraska - Lincoln. It has been accepted for inclusion in CSE Journal Articles by an authorized administrator of DigitalCommons@University of Nebraska - Lincoln. 


\section{Optical Communication Networks for the Next-Generation Internet}

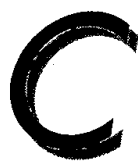

omputer and telecommunication networks are changing the world dramatically and will continue to do so in the foreseeable future. The Internet, primarily based on packet switches, provides very flexible data services such as e-mail and access to the World Wide Web. The Internet is a variable-delay, variable-bandwidth network that provides no guarantee on quality of service (QoS) in its initial phase. New services are being added to the pure data delivery framework of yesterday. Such high demands on capacity could lead to a "bandwidth crunch" at the core wide-area network, resulting in degradation of service quality. Fortunately, technological innovations have emerged which can provide relief to the end user to overcome the Internet's well-known delay and bandwidth limitations. At the physical layer, a major overhaul of existing networks has been envisaged from electronic media (e.g., twisted pair and cable) to optical fibers - in wide-area, metropolitan-area, and even local-area settings. In order to exploit the immense bandwidth potential of optical fiber, interesting multiplexing techniques have been developed over the years.

Wavelength-division multiplexing (WDM) is a very promising technique in which multiple channels are operated along a single fiber simultaneously, each on a different wavelength. These channels can be independently modulated to accommodate dissimilar bit rates and data formats, if so desired. Thus, WDM carves up the huge bandwidth of an optical fiber into channels whose bandwidths (1-10 $\mathrm{Gb} / \mathrm{s}$ ) are compatible with peak electronic processing speed. Optical fibers employing WDM can support around 1000 times the capacity of their electronic counterparts. WDM is already being deployed in commercial point-topoint fiber links, including undersea installations. WDMbased optical networks have been tested in the United States (MONET, NTONC projects), Europe (RACE, ACTS projects), and other countries. Several vendors have demonstrated WDM-based optical switching systems in the recent past.

Computer communication networks have evolved to make full use of the optical fiber technology. Paul E. Green, Jr. in his book Fiber Optic Networks (Prentice Hall,

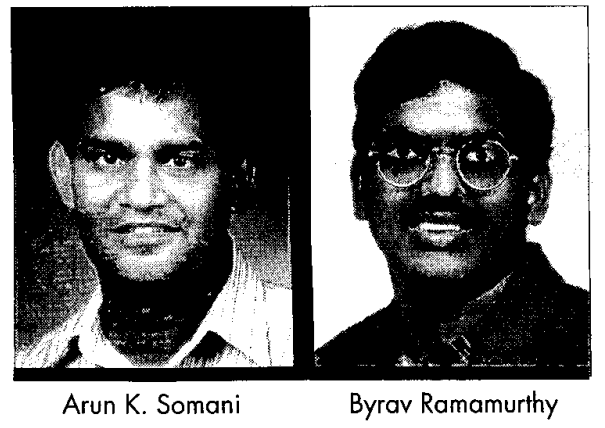

1993) refers to three generations in the evolution of highspeed networks. In a first-generation network, the nodes were interconnected with copper links which had limited bandwidth capabilities. In a second-generation network, optical fiber was used as a replacement for copper as the transmission medium, in view of its huge bandwidth capability. However, data sent over this network along multiple links undergoes optical-to-electronic conversion and vice versa at each intermediate node before reaching its destination. Hence, the network does not provide protocol transparency, that is, the capability to accommodate data comprising many different bit rates and formats at the same time. Data processing at each intermediate node also results in additional overhead. A third-generation network provides a continuous optical connection between all nodes. Data can be sent from one node to another entirely in the optical domain, providing complete transparency.

One may argue that both the second- and third-generation networks mentioned above can be referred to as optical networks since they use optical fiber as the transmission. medium. Note that the WDM technique can improve bandwidth availability in both configurations (providing WDM transmission in one and, additionally, WDM switching in the other). However, lately the term optical communication networks is increasingly used to refer to fiber-based networks which support high-bandwidth transmission supported by high-speed switching (either all optically or through electro-optical and opto-electronic conversions) and usually involving multiple wavelengths. Furthermore, recent advances in device technology have brought us closer than ever to realizing a full-fledged all-optical network over a large geographical domain.

Research in optical communication networks is not merely concerned with innovations at the physical layer. Optical networks provide the framework for supporting wavelength routing through the establishment of lightpaths, high-speed protection techniques at the optical layer, effective bandwidth management through dynamic setup and teardown of lightpaths, and elimination of intermediate protocol layers between user data and physical layer trans- 
mission. Design of architectures and protocols for these networks (using realistic assumptions about the capabilities of the underlying devices), evaluation of different routing, scheduling, protection, and multiplexing techniques, design and implementation of control and management platforms; and investigation of various IP over WDM scenarios are but a few of the research topics currently under investigation. Optical layer protection, traffic grooming, subwavelength utilization, and optical time-division multiplexing (OTDM) have been investigated for use in WDM optical networks in the last few years.

Through this special issue of IEEE Network, we are pleased to present a sampling of diverse facets of research in this exciting field.

The first article (by K. H. Liu, B. J. Wilson, and J. Y. Wei) presents a network management and visualization framework aimed at guiding the development of management applications for reconfigurable WDM optical networks. The authors share their experience in implementing the MONET network control and management system.

The next three articles are concerned with different aspects of providing fault-tolerant network connectivity in optical networks. The article by D. Zhou and S. Subramaniam presents a tutorial introduction to survivability techniques in both traditional and next-generation WDM optical networks.

The next article (by G. Mohan and C. Siva Ram Murthy) presents a survey of restoration schemes proposed in literature for providing fault tolerance to the optical layer in WDM-based transport networks.

The article by A. Fumagalli and L. Valcarenghi investigates the techniques available at both the IP and WDM layers in an optical network to support network survivability. The authors propose a heuristic technique to optimize the concurrent use of IP restoration and WDM protection schemes in the same network.

The last two articles in this issue investigate novel multiplexing and switching techniques suitable for next-generation optical networks. The article by A. Stok and E. H. Sargent provides a tutorial introduction to the technique of optical code-division multiple access (CDMA) and highlights directions for further research using this promising technique.

The article by S. Verma, H. Chaskar, and R. Ravikanth presents a framework to operate an IP network over WDM using a promising transmission technology - optical burst switching (OBS).

In bringing this special issue to you, we have had the generous support of the authors, the reviewers, the liaison editor, the Editor-in-Chief, and the publications staff. We thank them all for all their help and encouragement.

We hope you will enjoy reading the collection of articles in this special issue on Optical Communication Networks!

\section{Biography}

ARUN K. SOMANI [F] (arun@iastate.edu) is currently Nicholas Professor of Electrical and Computer Engineering at lowa State University. He earned his M.S.E.E. and Ph.D degrees in electrical engineering from McGill University, Montreal, Canada, in 1983 and 1985, respectively. He worked as scientific officer for the government of India, New Delhi, from 1974 to 1982. From 1985 to 1997 he was a faculty member at the University of Washington, Seattle, where he was a professor of electrical engineering and computer science and engineering from 1995 onward. His research interests are in the area of fault-tolerant computing, computer communication and networks, wireless and optical networking, computer archirecture, and parallel computer systems. He has taught courses in these areas and published more than 150 technical papers. He was the chief architect of the Proteus multicomputer system, designed and built at the University of Washington for coarse-grained parallel processing. He has served on several program committees of various conferences in his research areas, was General Chair of the IEEE Fault Tolerant Computing Symposium and Technical Program Chair of the IEEE Conference on Computer Communications and Networks. He is currently serving as an Associate Editor of IEEE Translations on Computers and an Editor of Microprocessors and Microsystems.

BYRAV RAMAMURTHY (byrav@cse.unl.edu) received his B.Tech, degree in computer science and engineering from the Indian Institute of Technology, Madras, in 1993. He received his MS and Ph.D. degrees in computer science from the University of Colifornia (UC) Davis in 1995 and 1998, respectively. Since August 1998 he has been an assistant professor in the Department of Computer Science and Engineering at the University of Nebraska-Lincoln (UNL). He is a founding codirector of the Advanced Networking and Distributed Experimental Systems (ANDES) Laboratory at UNL. He is the Feature Editor on Theses for Optical Network Magazine. He served as a member of the technical program committees for the IEEE GLOBECOM'99 Symposium on High Speed Networks, and the Eighth IEEE International Conference on Computer Communication and Network (ICCCN '99) and IEEE INFOCOM 2001. He was a recipient of the Indian National Talent Search scholarship and was a fellow of the Professors for the Future program at UC Davis. He is a recipient of the UNL Research Council Grant-in-Aid award for 1999 and the Layman Award for 2000. His research areas include optical networks, distributed systems, and telecommunications. His research on optical networks is funded by the National Science Foundation and Agilent Technologies. 